\title{
Emodin Successfully Inhibited Invasion of Brucella abortus Via Modulting Adherence, Microtubule Dynamics and ERK Signaling Pathway in RAW 264.7 Cells
}

\author{
Tran Xuan Ngoc Huy, Alisha Wehdnesday Bernardo Reyes, Huynh Tan Hop, Lauren Togonon Arayan, \\ Vu Hai Son, Wongi Min, Hu Jang Lee, and Suk Kim* \\ Institute of Animal Medicine, College of Veterinary Medicine, Gyeongsang National University, Jinju 52828, Republic of Korea
}

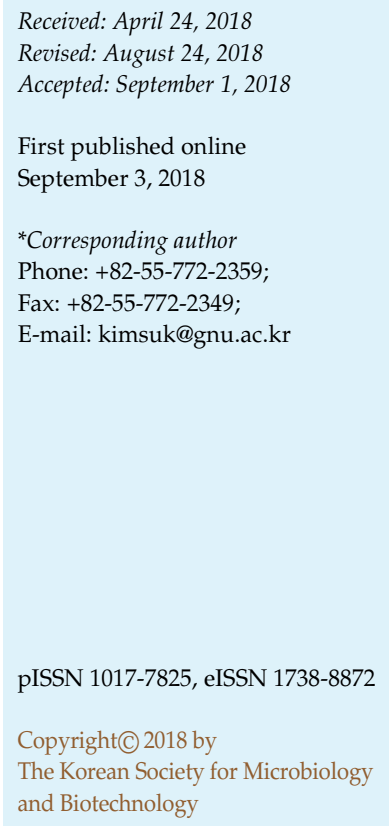

The aim of this work is to investigate the protective efficacy of emodin, an active, naturallyoccurring anthraquinone derivative of several traditional Chinese herbs, against Brucella abortus infection in macrophages. Brucella were incubated with different concentrations of emodin and showed that bacterial survival rates were markedly reduced in a dose-dependent manner at increasing incubation time points. Through bacterial infection assay, the highest non-cytotoxic concentration of emodin demonstrated attenuated invasion of Brucella into macrophages, however it did not inhibit the growth of these pathogens within the host cells. On the other hand, emodin effectively decreased the number of bacteria that adhered to host cells, which indicated its potential as an anti-adhesin agent. Furthermore, using immunoblotting and FACS assay for detecting MAPK signaling proteins and F-actin polymerization, respectively, the results showed that the emodin-incubated cells displayed modest reduction in the phosphorylation levels of ERK1/2 and inhibition of F-actin polymerization as compared to control cells. These findings indicate the potential use of emodin as a naturally-occurring alternative method for the prevention of animal brucellosis although this requires confirmation of safe clinical doses.

Keywords: Brucella abortus, emodin, antibacterial, invasion

\section{Introduction}

Brucella (B.) abortus is a facultative intracellular pathogen that resists killing by neutrophils, replicates within macrophages and persists as a chronic infection with diverse pathological manifestations including arthritis, endocarditis and meningitis in humans, and spontaneous abortion in domestic animals [1]. New cases of brucellosis in humans are reported to be approximately half a million annually, not reflecting the total number of cases worldwide since the disease remains undiagnosed or misdiagnosed [2].

The intracellular lifestyle of Brucella facilitates successful evasion of the host immune response and protection from antimicrobial compounds [3]. No approved human vaccine is available for brucellosis and the patients infected with Brucella require a prolonged course of treatment using multiple antimicrobial agents that are often harmful due to incidence of relapses as well as adverse effects that include hepatotoxicity and gastric damage [2]. Therefore, there is an urgent necessity for developing improved, safe and effective alternative treatment strategies for the disease.

Emodin (1,3,8-trihydroxy-6-methylanthraquinone), a naturally-occurring anthraquinone derivative and an active ingredient in traditional Chinese herbs Rheum palmatum, Polygonum cuspidatum, Polygonum multiflorum, Aloe vera and Cassia obtusifolia, has been demonstrated to possess a wide range of pharmacological benefits including antiviral, antibacterial, antiallergenic, antiosteoporotic, antidiabetic, anti-inflammatory and antitumor effects [4]. Despite these benefits of emodin, the compound has not been studied for its potential as an effective treatment for brucellosis. Consequently, the present study focuses on the effects of 
emodin on the survival of B. abortus, and the pathogen's ability to invade and subsequently replicate within a murine macrophage cell line of RAW 264.7 cells.

\section{Materials and Methods}

\section{Emodin Preparation}

Emodin was purchased from Sigma-Aldrich (USA). The powder was dissolved in dimethyl sulfoxide (DMSO) to the concentration of $1 \mathrm{mg} / \mathrm{ml}$ and then diluted to different concentrations in phosphate-buffered saline solution (PBS, pH 7.4).

\section{Bacterial Strain}

B. abortus 544 (ATCC 23448), a smooth, virulent B. abortus biovar 1 strain used in the present study, is a standard wild-type cultivated in Brucella broth or on agar (1.5\% agar) (Becton Dickinson, USA) and grown in broth at $37^{\circ} \mathrm{C}$ with shaking until the stationary phase was reached. Serial dilutions on agar plates were performed to assess the number of viable bacteria.

\section{Cell Culture}

RAW 264.7 cells (ATCC, USA) were grown at $37^{\circ} \mathrm{C}$ in $5 \% \mathrm{CO}_{2}$ atmosphere in RPMI 1640 containing 10\% (v/v) heat-inactivated fetal bovine serum (FBS) with or without antibiotics $(100 \mathrm{U} / \mathrm{ml}$ penicillin, and $100 \mu \mathrm{g} / \mathrm{ml}$ streptomycin) depending on the experiment. All the reagents were purchased from Gibco (USA). Fresh culture with PBS was used as a control in all in vitro assays performed.

\section{Cell Viability}

An overnight culture of RAW 264.7 cells at a concentration of $1 \times 10^{5}$ cells/well in a 96-well culture plate was incubated in the presence of emodin $(3,1.5,0.75,0.3$, and $0.15 \mu \mathrm{g} / \mathrm{ml})$ for $48 \mathrm{~h}$ and cell viability was evaluated using 3-(4,5-dimethylthiazol-2-yl)-2,5diphenyl-2H-tetrazolium bromide (MTT) cleavage assay as previously described [3]. The highest non-cytotoxic concentration of emodin $(0.3 \mu \mathrm{g} / \mathrm{ml})$ was used in the succeeding experiments.

\section{Bacterial Survival}

Brucella at a concentration of $1 \times 10^{4}$ colony-forming units $(\mathrm{CFU}) / \mathrm{ml}$ were added to different concentrations of emodin $(3,6$, and $15 \mu \mathrm{g} / \mathrm{ml}$ ) in PBS incubated at $37^{\circ} \mathrm{C}$ for $0,2,4,6$, and $24 \mathrm{~h}$. Bacterial survival rates were expressed as a percentage of the survival rate of the treated sample relative to the control sample set to $100 \%$.

\section{Bacterial Infection}

A bacterial invasion assay was performed as previously described [3]. Briefly, an overnight culture of RAW 264.7 cells at a concentration of $1 \times 10^{5}$ cells/well in a 96-well culture plate was pre-incubated with emodin or PBS for $4 \mathrm{~h}$. The cells were washed with PBS, incubated in fresh medium (RPMI 1640 with 10\% heatinactivated FBS) and then infected with B. abortus at multiplicities of infection (MOIs) of 10 . The cells were centrifuged at $150 \times \mathrm{g}$ for $10 \mathrm{~min}$ and incubated at $37^{\circ} \mathrm{C}$ in $5 \% \mathrm{CO}_{2}$ for 0 or $30 \mathrm{~min}$. At different time points, the cells were washed and incubated in fresh medium containing gentamicin $(30 \mu \mathrm{g} / \mathrm{ml})$ for $30 \mathrm{~min}$ to kill adhered and remaining extracellular bacteria. The cells were then washed again, lysed with distilled water, and the cell lysates were spread onto Brucella agar plates in triplicate. In a separate procedure for intracellular replication assay, cells were infected with B. abortus, washed and then incubated with fresh medium containing gentamicin and emodin or PBS for 2, 24, and $48 \mathrm{~h}$. Bacterial infection, washing, lysing and plating procedures were done in the same manner as in the invasion assay.

\section{Bacterial Adherence}

An overnight culture of RAW 264.7 cells at a concentration of $1 \times 10^{6}$ cells/well in a 12-well culture plate with 18 -mm diameter glass coverslips (Fisher Scientific, USA) was pre-incubated with emodin or PBS for $4 \mathrm{~h}$. Forty minutes before infection, the cells were treated with cytochalasin D $(500 \mu \mathrm{g} / \mathrm{ml})$ to inhibit bacterial internalization. Infection of cells for $30 \mathrm{~min}$ was done in the same manner as in the bacterial invasion assay. After washing, cells were fixed with $4 \%$ paraformaldehyde at $37^{\circ} \mathrm{C}$ for $30 \mathrm{~min}$, permeabilized with cold methanol $\left(-20^{\circ} \mathrm{C}\right)$ for $10 \mathrm{sec}$, incubated with anti-B. abortus polyclonal rabbit serum $(1: 500)$ at $37^{\circ} \mathrm{C}$ for $1 \mathrm{~h}$ and then with fluorescein isothiocyanate (FITC)-conjugated goat anti-rabbit immunoglobulin G (IgG) (1:500, Sigma-Aldrich, USA) at $37^{\circ} \mathrm{C}$ for $1 \mathrm{~h}$. Random selection of one-hundred macrophages was done to determine the number of bacteria that adhered to these cells.

\section{Fluorescence Microscopy}

RAW 264.7 cells were prepared and incubated with emodin or PBS as in the bacterial adherence assay. In parallel, negative control cells were prepared for comparison. After washing, cells were infected with FITC (Sigma-Aldrich, USA)-labelled B. abortus for $10 \mathrm{~min}$ as previously described [3]. The cells were then fixed, permeabilized with $0.1 \%$ Triton X-100 for 10 min, blocked with $2 \%$ goat serum in PBS for $30 \mathrm{~min}$ and stained with $0.1 \mu \mathrm{M}$ rhodaminephalloidin (Cytoskeleton, Inc., USA) for $30 \mathrm{~min}$ at $22^{\circ} \mathrm{C}$. A laser scanning confocal microscope (Olympus FV1000, Japan) and FV10-ASW Viewer 3.1 software were used to capture and process fluorescence images.

\section{Flow Cytometry}

An overnight culture of RAW 264.7 cells at a concentration of $1 \times 10^{8}$ cells/well in a 6-well culture plate was pre-incubated with emodin or PBS for $4 \mathrm{~h}$. A similar procedure was done as previously described [3]. The F-actin content was then measured using a BD FACSVerse flow cytometer (BD Biosciences, USA).

\section{Western Blot}

RAW 264.7 cells were prepared and pre-incubated with emodin or PBS as in the flow cytometry for F-actin content determination. 
After washing, the cells were incubated at $4^{\circ} \mathrm{C}$ overnight using ice-cold radioimmunoprecipitation assay (RIPA) buffer with $1 \%$ protease inhibitor cocktail. The cell lysates were collected and the protein concentration was measured using the Bradford protein assay (Bio-Rad, USA). Proteins were separated by SDS-PAGE and electrically transferred onto Immobilon-P membranes (Millipore, USA). The membranes were incubated with mitogen-activated protein kinases (MAPKs) (ERK1/2, JNK and p38 $\alpha$ ) as previously described [3], exposed to a Molecular Imager ChemiDoc XRS+ system machine (Bio-Rad, USA) and the immunoblot signals were quantified using NIH ImageJ software. In addition, we determined the effect of ERK activator, MEK-1 (Santa Cruz Biotechnology, Inc., Germany) on the internalization of Brucella in emodin-incubated cells. The same procedures were performed as in the bacterial invasion assay wherein after pre-incubation of macrophages with

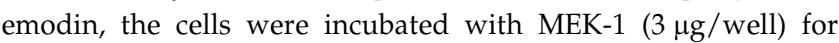
$45 \mathrm{~min}$ prior to infection.

\section{Statistical Analysis}

The results are expressed as the means with standard deviation (SD) for replicate experiments. Statistical analysis was performed using GraphPad InStat software version 3 (GraphPad Software, Inc., USA). Student's $t$-test or one-way ANOVA was used to make statistical comparisons between the groups. Differences of $p<0.05$ were considered to be statistically significant.

\section{Results}

\section{Effects on Cell Viability and Bacterial Survivability}

RAW 264.7 cells were incubated with different concentrations of emodin $(3,1.5,0.75,0.3,0.15 \mu \mathrm{g} / \mathrm{ml})$ for $48 \mathrm{~h}$. The OD value (cell survival) at higher concentrations

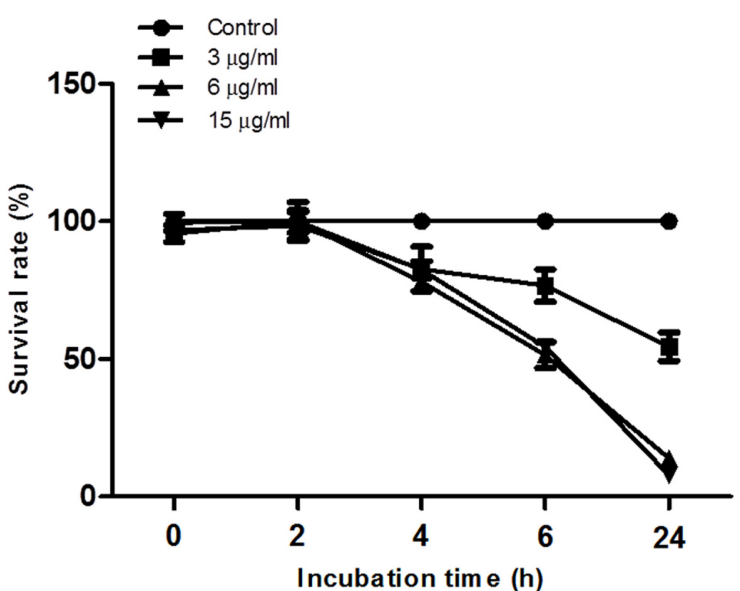

Fig. 1. Incubation of B. abortus with different concentrations of emodin $(0,3,6$, and $15 \mu \mathrm{g} / \mathrm{ml})$ at $0,2,4,6$, and $24 \mathrm{~h}$. Data represent mean $\pm \mathrm{SD}$ of at least two replicates.

$(3,1.5$, and $0.75 \mu \mathrm{g} / \mathrm{ml})$ decreased, which indicated cytotoxic effect. The highest non-cytotoxic concentration $(0.3 \mu \mathrm{g} / \mathrm{ml})$ without significant effect on the cell viability was used in the succeeding experiments. In addition, survival rates of Brucella were reduced significantly in higher concentrations of emodin $(3,6$, and $15 \mu \mathrm{g} / \mathrm{ml})$ over increasing time of incubation (Fig. 1), indicating that emodin has antibacterial effect against Brucella in a dose-dependent manner.

\section{Effects on Bacterial Invasion and Intracellular Growth}

Invasion of Brucella into macrophages was determined at

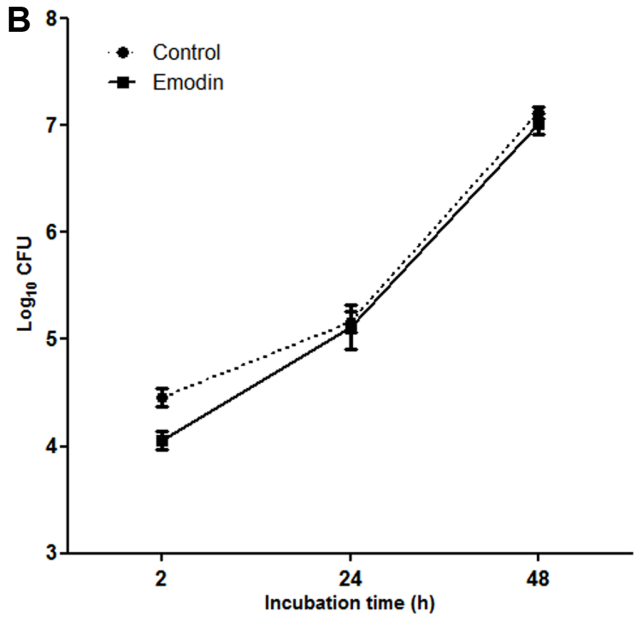

Fig. 2. The effects of emodin on the efficiency of (A) bacterial invasion at 0 and $30 \mathrm{~min}$ post-infection time and (B) intracellular replication of $B$. abortus at 2,24 , and $48 \mathrm{~h}$ post-incubation.

Data represent the mean \pm SD of at least two replicates. Statistically significant differences relative to the untreated control are indicated by asterisks $\left({ }^{*} p<0.05 ; * * p<0.01\right)$. 
indicated times after pre-incubation with emodin. The number of CFU was markedly reduced in emodin-incubated cells as compared to control cells at $0(p<0.05)$ and $30 \mathrm{~min}$ $(p<0.01)$ post-infection (Fig. 2A). However, this pattern was not evident in the intracellular growth of Brucella within macrophages where the number of CFU in the emodin-incubated cells was not significantly different from that of the control cells (Fig. 2B). These data indicated that emodin has an inhibitory effect on the phagocytosis of Brucella into macrophages but no influence on the bacterial replication when the pathogens are inside the host cells.

\section{Effects on Bacterial Phagocytosis and Adherence}

Since emodin significantly inhibited the entry of Brucella into macrophages, we evaluated its effect on actin polymerization, which is involved in the phagocytic uptake of microbes. In this study, the fluorescence microscopy examinations revealed reduced formation of lamellipodia, filopodia and microspikes in emodin-incubated cells as compared to control cells after exposure to Brucella (Fig. 3A), indicating an inhibition in the F-actin polymerization in these cells and this was confirmed by quantification of Factin content using flow cytometry. The results showed a significant reduction in the F-actin content in emodinincubated cells as compared to control cells (Fig. 3B). On the other hand, we assessed the involvement of emodin on the pathogen's adherence to host cells by fluorescence microscopy. In the emodin-incubated cells, the number of bacteria adhered was significantly reduced $(34 \pm 7.5)$ as compared to control cells $(81 \pm 11.09)$, showing a reduction rate of $58.07 \pm 9.8 \% \quad(p<0.05)$ (Fig. 4$)$. Taken together, these findings indicated that emodin attenuated F-actin polymerization, which is a concern in the phagocytosis of Brucella in macrophages [5], and effectively attenuated attachment of these pathogens on the surface membrane of macrophages that could explain the reduction in the
A
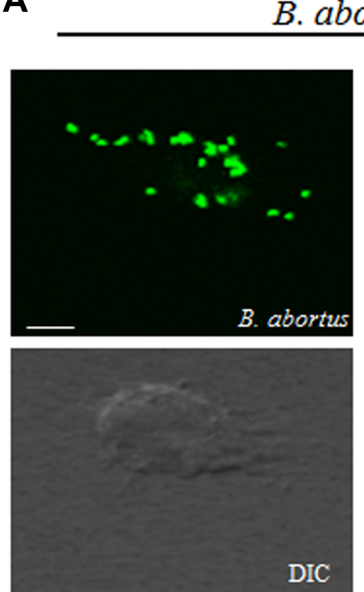
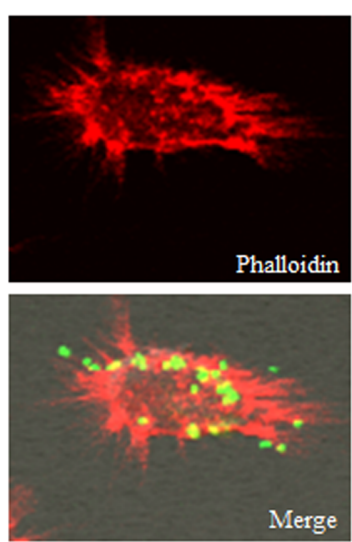

Emodin + B. abortus
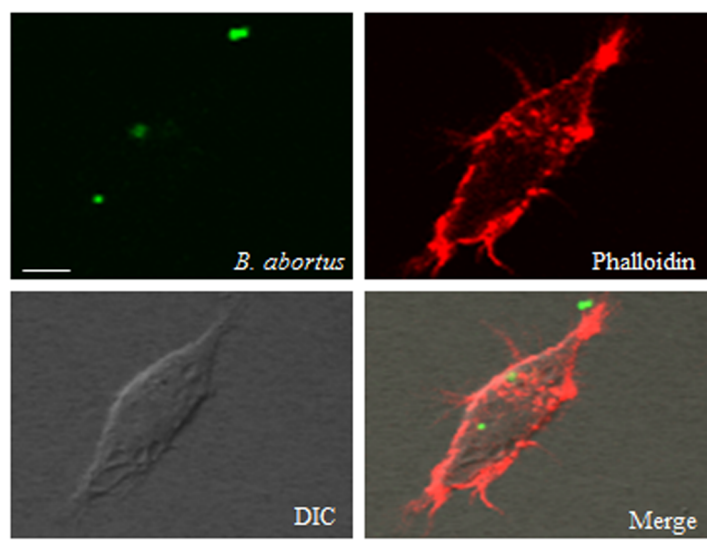

B

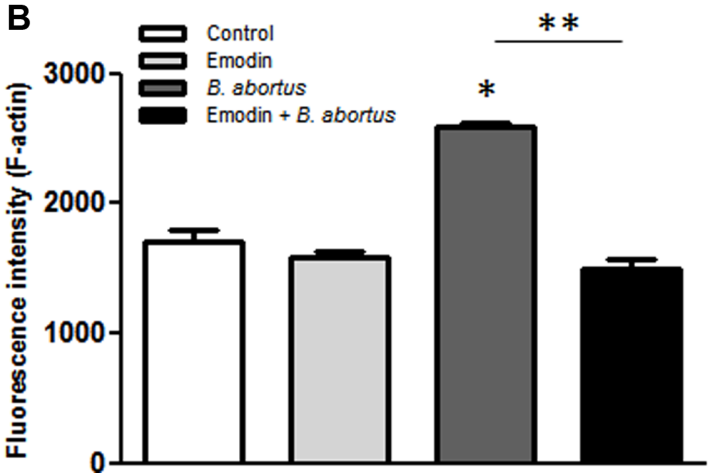

Fig. 3. The effects of emodin on the modulation of F-actin polymerization using (A) fluorescence microscopy (scale bars $=5 \mu \mathrm{m})$ and (B) flow cytometry analysis.

Data represent mean \pm SD of at least two replicates. Statistically significant differences relative to the untreated control are indicated by asterisks $\left({ }^{*} p<0.05 ;{ }^{* *} p<0.01\right)$. 


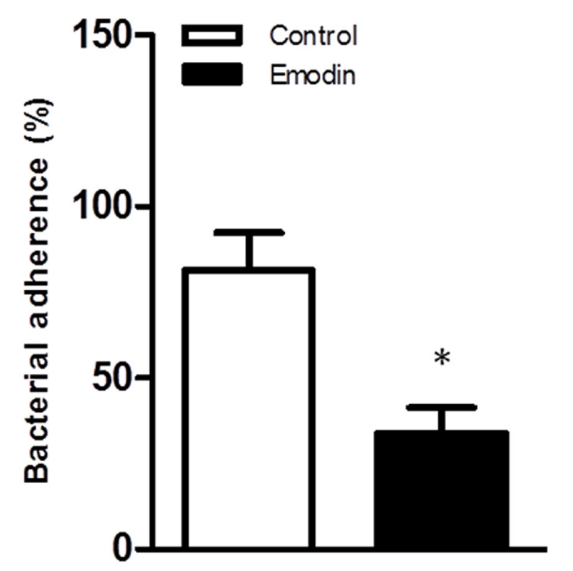

Fig. 4. The effects of emodin on the adherence of B. abortus in RAW 264.7 cells.

Data represent mean \pm SD of at least two replicates. Statistically significant differences relative to the untreated control are indicated by asterisks $\left({ }^{*} p<0.05\right)$.

phagocytosis of Brucella.

\section{Effects on Phagocytic Signals}

Mitogen-activated protein kinase (MAPK) plays an important role in the phagocytic microbial entry mechanism including phagocytosis and actin cytoskeleton remodeling $[5,6]$. The phosphorylation level of ERK1/2 in emodinincubated cells at $30 \mathrm{~min}$ post-infection was reduced by 1.29-fold compared to control cells (Fig. 5). These findings suggested that the inhibitory effects of emodin on the phagocytosis of Brucella and F-actin polymerization in RAW 264.7 cells could be due to restraint of MAPKs, particularly ERK1/2.

Since emodin exclusively repressed phosphorylation of ERK1/2 and did not affect phosphorylations of JNK and p38, we treated emodin-incubated cells with ERK activator (MEK-1) to determine the effects in the invasion of Brucella in these cells. The results showed that internalization of Brucella was increased at $0(p<0.01)$ and $30 \mathrm{~min}(p<0.05)$ post-infection as compared to emodin-incubated cells, although slightly decreased compared with the PBSincubated cells but not significantly (Fig. 2A), which suggested that the mechanisms involved in the attenuation of bacterial invasion by emodin could be ERK-dependent.

\section{Discussion}

Brucella spp. are considered as major veterinary pathogens that can cause serious disease in humans with annually
A
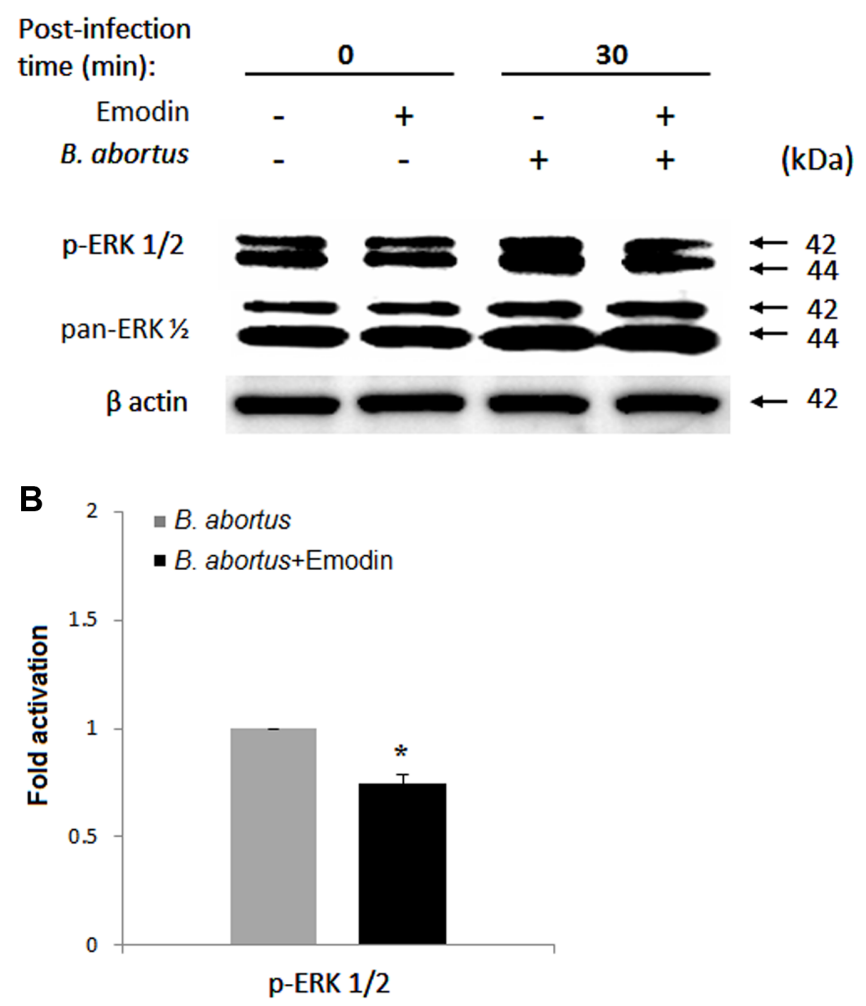

Fig. 5. The effects of emodin on the phosphorylation level of ERK1/2 signaling in RAW 264.7 cells.

(A) Western blot images and (B) the phosphorylation levels of ERK1/ 2 shown are representative of at least three independent experiments. Statistically significant differences relative to the untreated control are indicated by asterisks $\left({ }^{*} p<0.05\right)$.

reported new cases of about 500,000 around the globe and yet no patient-friendly treatment or effective vaccines are available for humans [7, 8]. These pathogens undergo several interactions with host cells as they invade and replicate within professional phagocytic cells, undertaking evasion of the killing mechanisms within macrophages, modulation of host immune response and dissemination to preferred tissues through cellular tropism - leading to chronicity and a difficult-to-treat form of infection $[9,11]$. Currently, treatment of brucellosis using traditional plants and natural products has been massively reported as effective alternative control of this disease. The previous study proved that the Chinese herbs such as Coptis chinensis, Radix paeoniae rubra, Galla chinensis, and Cortex phellodendri possessed inhibitory effect against Brucella melitensis strains [10]. Moreover, the study by Lee (2011) elucidated that Galla Rhois, which has long been applied in traditional Korean and Oriental medicine, showed potential for use in 
the prevention and treatment of brucellosis while also stating that some components of Galla Rhois had inhibitory effects on the growth of Clostridium spp., Escherichia coli, and Salmonella typhimurium [12].

Most bacterial mechanisms for engaging with host cells strictly depend on their adhesion to host cells [13]. Brucellae are known to bind to distinct phagocytic or unknown receptors in macrophages that subsequently lead to zipperlike phagocytosis or through lipid raft microdomains requiring F-actin polymerization and dynamic rearrangement of the actin cytoskeleton - which are essential in the uptake of microbes [9]. Since there is an alarming increase in drugresistant bacteria and bacterial adhesion is one of the initial stages of infectious processes [14], one attractive approach is the use of natural agents that can effectively interfere with the ability of these pathogens to adhere to target cells. Emodin has been shown to significantly inhibit formation of biofilm in Pseudomonas aeruginosa and Stenotrophomonas maltophilia, likely via penetration into the biofilm and interference with the quorum sensing (QS) system, leading to bacterial detachment and dispersal from the surface [15]. Furthermore, emodin has been reported to disrupt lipid rafts in the membrane of CD14-negative endothelial cells by depleting cholesterol [16]. Similarly, our results showed that emodin effectively inhibited the adherence of Brucella into the surface membrane of macrophages probably due to its ability to disrupt lipid rafts, or through competition of common receptors like those of Brucella, although this needs to be proven.

The antibacterial efficacy of emodin has been demonstrated against several microorganisms including Bacillus subtilis, Staphylococcus aureus, Helicobacter pylori and Haemophilus parasuis [17-20]. It has been observed that the antibacterial activity of emodin was concentration-dependent and attributed to its ability to destroy cell membrane integrity, increase membrane permeability and DNA damage [20]. Similarly, the antibacterial efficacy of emodin was observed in a concentration-dependent manner at increasing incubation periods. These results suggest that emodin is a viable candidate for treating animal brucellosis although it requires systematic animal toxicology experiments for the confirmation of safe clinical doses.

F-actin polymerization, which is an essential part of the entry mechanisms in phagocytosis of pathogens, has been identified to be involved in the ingestion of Brucella in epithelial cells and macrophages [21]. Emodin was reported to markedly inhibit the epidermal growth factor-induced formation of filopodia and lamellipodia [22]. This is similar to our present study in which the incubation of RAW 264.7 cells with emodin resulted in reduced formation of filopodial structures and F-actin content upon exposure to Brucella. On the other hand, the activation of the MAPK cascade also plays an important role in the phagocytosis of bacteria and remodeling of the actin cytoskeleton [13,23]. The effect of emodin on MAPK signaling pathways was previously investigated and showed that it did not affect the activation of MAPK p38 and JNK but markedly inhibited the activation of ERK1/2 in rat C6 glioma cell line [24]. Furthermore, emodin effectively attenuated phosphorylation of ERK in HepG2 cells [25]. These results were in agreement to our present study where incubation of RAW 264.7 cells with emodin notably inhibited ERK1/2 phosphorylation levels. This suggests that the inhibitory effect of emodin on Brucella invasion into macrophages could be attributed to downregulaton of MAPK. In conclusion, emodin provides protection against Brucella invasion into macrophages, suggesting that the use of emodin, possibly as a feed supplement, may provide a natural alternative option in the prevention of animal brucellosis although further validation of its therapeutic use in animals is still required.

\section{Acknowledgment}

This work was supported by a grant from the Korea Health Technology R\&D Project through the Korea Health Industry Development Institute (KHIDI) funded by the Ministry of Health \& Welfare, Korea (HI16C2130).

\section{Conflict of Interest}

The authors have no financial conflicts of interest to declare.

\section{References}

1. Campos MA, Rosinha GMS, Almeida IC, Salgueiro XS, Jarvis BW, Splitter GA, et al. 2004. Role of Toll-like receptor 4 in induction of cell-mediated immunity and resistance to Brucella abortus infection in mice. Infect. Immun. 72: 176-186.

2. Czyz DM, Jain-Gupta N, Shuman HA, Crosson S. 2016. A dual-targeting approach to inhibit Brucella abortus replication in human cells. Sci. Rep. 6: 35835.

3. Reyes AWB, Arayan LT, Simborio HL, Hop HT, Min W, Lee HJ, et al. 2016. Dextran sulfate sodium upregulates MAPK signaling for the uptake and subsequent intracellular survival of Brucella abortus in murine macrophages. Microb. Pathog. 91: 68-73.

4. Wei G, Wu Y, Gao Q, Zhou C, Wang K, Shen C, et al. 2017. Effect of emodin on preventing postoperative intra-abdominal 
adhesion formation. Oxid. Med. Cell Longev. 2017: 1-12.

5. Lee JJ, Kim DH, Kim DG, Lee HJ, Min W, Rhee MH, et al. 2012. Phellinus baumii extract influences pathogenesis of Brucella abortus in phagocyte by disrupting the phagocytic and intracellular trafficking pathway. J. Appl. Microbiol. 114: 329-338.

6. Reyes AW, Kim DG, Simborio HL, Hop HT, Arayan LT, Min W, et al. 2016. Methyl gallate limits infection in mice challenged with Brucella abortus while enhancing the inflammatory response. J. Appl. Microbiol. 120: 552-559.

7. Jimenez de Bagues MP, Gross A, Terraza A, Dornand J. 2005. Regulation of the mitogen-activated protein kinases by Brucella spp. expressing a smooth and rough phenotype: relationship to pathogen invasiveness. Infect. Immun. 73: 3178-3183.

8. Ahmed W, Zheng K, Liu ZF. 2016. Establishment of chronic infection: Brucella's stealth strategy. Front. Cell Infect. Microbiol. 6: 1-12.

9. Lee JJ, Kim DH, Kim DG, Lee HJ, Min W, Rhee MH, et al. 2013. Toll-like receptor 4-linked Janus kinase 2 signaling contributes to internalization of Brucella abortus by macrophages. Infect. Immun. 81: 2448-2458.

10. Wen KW, Bejo SK. 2010. Screening of Chinese medicinal herbs for the inhibition of Brucella melitensis. $5^{\text {th }}$ Proceedings of the Seminar in Veterinary Sciences.

11. de Figueiredo P, Ficht TA, Rice-Ficht A, Rossetti CA, Adams LG. 2015. Pathogenesis and immunobiology of brucellosis: review of Brucella-host interactions. Am. J. Pathol. 185: 1505-1517.

12. Lee JJ, Bae JH, Kim DH, Lim JJ, Kim DG, Lee HJ, et al. 2011. Intracellular replication inhibitory effects of Galla Rhois ethanol extract for Brucella abortus infection. J. Ethnopharmacol. 138: 602-609.

13. Stones DH, Krachler AM. 2016. Against the tide: the role of bacterial adhesion in host colonization. Biochem. Soc. Trans. 44: $1571-1580$

14. Ofek I, Hasty DL, Sharon N. 2003. Anti-adhesion therapy of bacterial diseases: prospects and problems. FEMS Immunol.
Med. Microbiol. 38: 181-191.

15. Rabin N, Zheng Y, Opoku-Temeng C, Du Y, Bonsu E, Sintim HO. 2015. Agents that inhibit bacterial biofilm formation. Future Med. Chem. 7: 647-671.

16. Meng G, Liu Y, Lou C, Yang H. 2010. Emodin suppresses lipopolysaccharide-induced pro-inflammatory responses and NF- $\mathrm{KB}$ activation by disrupting lipid rafts in CD14-negative endothelial cells. Br. J. Pharmacol. 161: 1628-1644.

17. Wang HH, Chung JG. 1997. Emodin-induced inhibition of growth and DNA damage in the Helicobacter pylori. Curr. Microbiol. 35: 262-266.

18. Chukwujekwu JC, Coombes PH, Mulholland DA, van Staden J. 2006. Emodin, an antibacterial anthraquinone from the roots of Cassia occidentalis. S Afr. J. Bot. 72: 295-297.

19. Liu M, Peng W, Qin R, Yan Z, Cen Y, Zheng X, et al. 2015. The direct anti-MRSA effect of emodin via damaging cell membrane. Appl. Microbiol. Biotechnol. 99: 7699-7709.

20. Li L, Song X, Yin Z, Jia R, Li Z, Zhou X, et al. 2016. The antibacterial activity and action mechanism of emodin from Polygonum cuspidatum against Haemophilus parasuis in vitro. Microbiol. Res. 186-187: 139-145.

21. Pei J, Turse JE, Ficht TA. 2008. Evidence of Brucella abortus OPS dictating uptake and restricting NF-kB activation in murine macrophages. Microbes Infect. 10: 582-590.

22. Huang Q, Shen HM, Ong CN. 2005. Emodin inhibits tumor cell migration through suppression of the phosphatidylinositol 3-kinase-Cdc42/Rac1 pathway. Cell Mol. Life Sci. 62: 1167-1175.

23. Shan L, He P, Sheen J. 2007. Intercepting host MAPK signaling cascades by bacterial type III effectors. Cell Host Microbe 1: 167-174.

24. Mijatovic S, Maksimovic-Ivanic D, Radovic J, Miljkovic D, Harhaji L, Vuckovic O, et al. 2005. Anti-glioma action of aloe emodin: the role of ERK inhibition. Cell Mol. Life Sci. 62: 589-598.

25. Cui Y, Lu P, Song G, Liu Q, Zhu D, Liu X. 2016. Involvement of PI3K/Akt, ERK and p38 signaling pathways in emodin-mediated extrinsic and intrinsic human hepatoblastoma cell apoptosis. Food Chem. Toxicol. 92: 26-37. 